\title{
Escala de Avaliação do Comportamento Alimentar no Transtorno do Espectro Autista: estudo de validação
}

\author{
The Scale for Evaluating Eating Behaviour in Autism \\ Spectrum Disorder: validation study \\ Cristiane Pinheiro Lázaro ${ }^{1,2}$ \\ https://orcid.org/0000-0002-0127-0227 \\ Gustavo Marcelino Siquara² \\ https://orcid.org/0000-0002-4495-6835 \\ Milena Pereira Pondé2 \\ https://orcid.org/0000-0002-1292-5487
}

\section{Palavras-chave}

Comportamento alimentar, TEA, escala de avaliação, autismo.

\section{RESUMO}

Objetivo: O objetivo do estudo foi construir os itens e realizar a validade de conteúdo e construto da Escala de Comportamento Alimentar do Autismo. Métodos: Uma equipe multidisciplinar analisou a validade do conteúdo. A escala foi aplicada de forma verbal e individualizada a pais de pessoas com transtorno do espectro autista (TEA) para ajuste semântico. Essa primeira versão da escala foi respondida por 298 pessoas, sendo feita uma análise dos componentes principais com uma rotação Varimax. Resultados: Dos 53 itens inicialmente desenvolvidos para o estudo do construto, 33 mostraram-se válidos para a avaliação do atributo e três foram acrescentados, compondo a segunda versão da escala, que foi respondida por 130 pessoas. Dos 35 itens que permaneceram após a primeira análise fatorial, 26 mostraram-se válidos para a avaliação do atributo e foram distribuídos em sete dimensões: motricidade na mastigação, seletividade alimentar, habilidades nas refeições, comportamento inadequado relacionado às refeições, comportamentos rígidos relacionados à alimentação, comportamento opositor relacionado à alimentação, alergias e intolerância alimentar. A estrutura final da escala ficou composta por 26 itens, distribuídos em sete fatores, apresentando um valor geral de confiabilidade de 0,867. Conclusão: A escala visa identificar as dimensões do comportamento alimentar que se encontram alteradas, proporcionando um direcionamento mais específico em relação à terapêutica, podendo também ser utilizada para mensurar a evolução do tratamento.

\section{ABSTRACT}

Objective: An evaluation instrument was developed to assess the eating behavior of individuals with autism spectrum disorder (ASD), both from the professional viewpoint and based on aspects identified as problematic by parents at semi-structured interviews. Methods: A multidisciplinary team analyzed content validity. The scale was applied verbally and individually to parents of individuals with ASD for semantic adjustment. Overall, 298 individuals

1 Escola BAHIANA de Medicina e Saúde Pública, Laboratório Interdisciplinar de Pesquisa em Autismo (LABIRINTO), Salvador, BA, Brasil. 2 Universidade Federal da Bahia (UFBA); Escola BAHIANA de Medicina e Saúde Pública, Laboratório Interdisciplinar de Pesquisa em Autismo (LABIRINTO), Salvador, BA, Brasil.

7/Out/2019

Aprovado em

24/Nov/2019

DOI: 10.1590/0047-2085000000246

Endereço para correspondência: Cristiane Pinheiro Lázaro

Escola BAHIANA de Medicina

e Saúde Pública, Laboratório

Interdisciplinar de Pesquisa em Autismo

Av. Dom João VI Avenue, 295

40290-000 - Salvador, BA, Brasil

Telefone: (71) 2101-1901

E-mail: lazarocris@hotmail.com 


\section{Keywords}

Eating behavior, ASD, evaluation scale, autism. answered this preliminary version of the scale, with varimax rotation being used to analyze the principal components. Results: Of the 53 items initially developed to assess the construct, 33 proved valid for evaluation of the attribute and three were added, thus obtaining the second version of the scale, which was then answered by 130 individuals. Of the 35 items that remained after the first factor analysis, 26 proved valid for evaluation of the attribute and were divided into seven domains: the motricity of chewing, food selectivity, mealtime skills, inappropriate mealtime behavior, inflexible eating-related behavior, hostility towards food, and food allergies and intolerance. The final structure of the scale was composed of 26 items, distributed in seven factors, presenting an overall reliability value of 0.867 . Conclusion: The scale aims to identify the areas of eating behavior that are altered in individuals with ASD, allowing more specific therapy to be implemented. The tool could also be used to measure the effectiveness of treatment.

\section{INTRODUÇÃO}

Pesquisas que investigaram os problemas alimentares em pessoas com transtorno do espectro autista (TEA) indicam que uma parcela dessa população apresenta inabilidades motoras orais relacionadas à mastigação e à deglutição', problemas no trato gastrointestinal (TGI)² e disfunção sensorial2,3. Os problemas relacionados à modulação sensorial, na forma hipo ou hiper-reativa, interferem diretamente no paladar, olfato, audição, visão, tato, sistema vestibular e propriocepção4, de tal modo, que é razoável supor que esses componentes podem influenciar de forma direta ou indireta os problemas comportamentais e alimentares citados anteriormente.

Com o objetivo de investigar o comportamento alimentar dos indivíduos com TEA, pesquisadores elaboraram escalas que pudessem ser preenchidas por pais ou cuidadores. Essas escalas foram elaboradas para identificar informações relativas à manutenção e ao agravamento do comportamento alimentar. Contudo, esses instrumentos se basearam no Diagnostic and Statistical Manual of Mental Disorders $(\mathrm{DSM}-\mathrm{IV})^{5}$ para o diagnóstico e não levaram em consideração as queixas alimentares relatadas pelos cuidadores. Sob a epígrafe de "Transtornos Invasivos do Desenvolvimento", o DSM-IV listava o autismo, o transtorno desintegrativo da infância, a síndrome de Asperger, a síndrome de Rett e o transtorno invasivo do desenvolvimento sem outra especificação. No entanto, o DSM-56 reflete a visão científica de que aqueles transtornos (exceto a síndrome de Rett e o transtorno desintegrativo) são na verdade a mesma condição e que o transtorno do espectro do autismo possui gravidade sintomática distinta em relação ao déficit na comunicação, interação social e padrão de comportamentos, interesses e atividades restritas e repetitivas.

$\mathrm{O}$ aspecto comum a todos os instrumentos encontrados na literatura é que o ponto de partida para as questões formuladas é sempre a experiência clínica dos profissionais que elaboram a escala ou a literatura científica sobre o tema. Nenhum dos estudos aborda o que os pais de pessoas com TEA consideram como problemático no comportamento alimen- tar desses indivíduos. Consideramos que uma avaliação mais completa do comportamento alimentar deve incluir a visão profissional e também os aspectos identificados pelos pais, na prática cotidiana com essas crianças. Não existe até o momento nenhum instrumento desenvolvido com a população brasileira que busque avaliar o comportamento alimentar de pessoas com TEA. Por isso, o objetivo de desenvolver um instrumento e realizar a validade de conteúdo e construto da Escala de Comportamento Alimentar do Autismo.

\section{MÉTODOS}

O fluxograma descrito na figura 1 detalha o processo de elaboração da escala, que foi dividido em três fases.

\section{Fase 1}

Para a primeira versão da escala, foi feita uma ampla revisão da literatura sobre o tema e uma série de entrevistas com pais de pessoas com TEA. Foi feita uma revisão sistemática
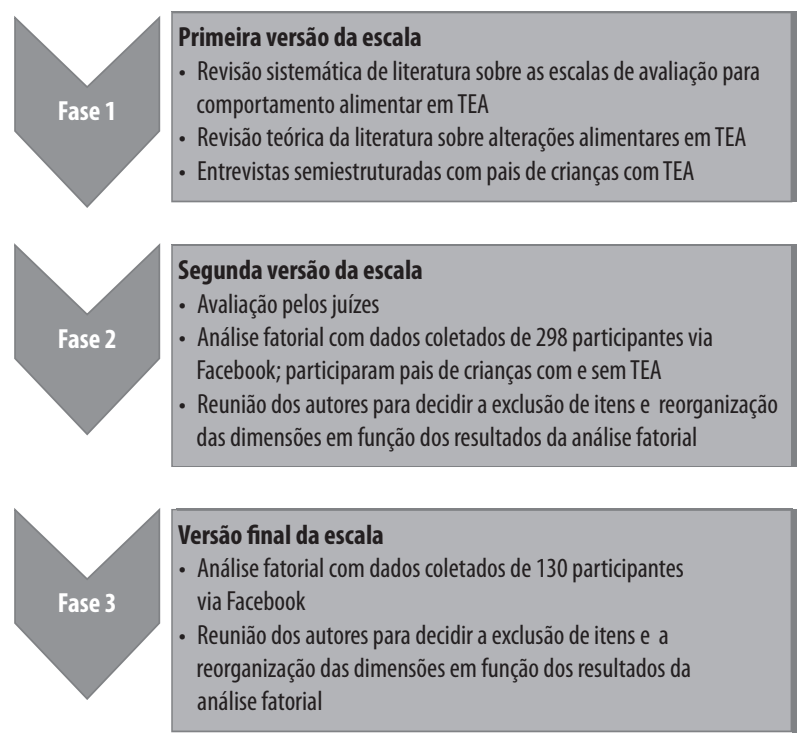

Figura 1. Fluxograma com as fases da elaboração da escala. 
da literatura buscando os instrumentos de avaliação já existentes em outras línguas, com o fito de identificar os aspectos positivos deles e suplantar as falhas dos instrumentos já estabelecidos? ${ }^{7}$. A revisão teórica teve como objetivo identificar as alterações alimentares já descritas em pessoas com TEA. Buscando acrescentar à literatura científica vigente aspectos que os pais consideram problemáticos no padrão alimentar dos seus filhos, foram feitas entrevistas semiestruturadas com mães de pessoas com TEA, para identificar o que elas consideravam como problema em relação ao comportamento alimentar dos seus filhos. A metodologia completa e o resultado desse estudo está disponível para consulta em publicação préviå

\section{Fase 2}

A primeira versão da escala foi submetida à avaliação por juízes e aplicada a pais de pessoas com e sem TEA, gerando uma segunda versão. O foco dessa fase foi determinar os itens que possuem consistência interna e que são clinicamente relevantes em termos de validade de conteúdo, e dividi-los em dimensões internamente válidas. A validade do conteúdo foi alcançada inicialmente por meio dos conhecimentos clínicos dos autores que construíram os itens da escala inicial. Após a elaboração da primeira versão da escala, esta foi submedida à análise da validade do conteúdo por uma equipe multidisciplinar de avaliadores (dois neuropediatras, três fonoaudiólogos, um terapeuta ocupacional, um psicopedagoga, dois psicólogos), todos com experiência clínica de pelo menos cinco anos no atendimento de pessoas com TEA.

A escala foi projetada com o objetivo de ser preenchida por pais e cuidadores, sem o auxílio dos pesquisadores. A desvantagem dessa abordagem é que ninguém está disponível para ajudar o entrevistado9. Para minimizar esse problema, foi realizada, de forma individual, a análise semântica da escala com um grupo de 20 pais cujos filhos são acompanhados em uma instituição especializada no atendimento psicopedagógico de pessoas com TEA. Foram registradas as dúvidas quanto à compreensão da escala, bem como as sugestões para melhorar a compreensão do texto, quando lido sem auxílio.

O processo de validação de um instrumento de mensuração de um constructo psicológico requer a coleta de diversas evidências empíricas ${ }^{10}$. A validade se refere a um julgamento em relação ao grau em que a evidência empírica e racionalizações teóricas apoiam as inferências baseadas em escores de testes ou escalas ${ }^{11}$. Nesse sentido, buscou-se analisar evidências de validade de construto e a estrutura fatorial da Escala de Comportamento Alimentar. Para a coleta de dados, com vistas à validação e à normatização da escala, os itens da primeira versão da escala foram digitados em um formulário de enquetes do Facebook, publicado na linha do tempo de profissionais que trabalham como autismo e no grupo dos pesquisadores da linha de pesquisa (o grupo reúne profissionais e pais de indivíduos com TEA). Foi solicitado que pais de pessoas com ou sem TEA respondessem ao questionário, após lerem e aceitarem o termo de consentimento.

Foram examinadas as propriedades psicométricas da escala por meio da consistência interna dos fatores aplicando a análise fatorial exploratória, para examinar a estrutura dos fatores para os dados atuais. Os padrões de fatores foram girados com rotação Varimax. Cargas de fator $>0,4$ foram consideradas de grande ou moderada importância, sendo, portanto, consideradas significativas ${ }^{12}$. A análise fatorial, juntamente com a consistência interna em termos de alfa de Cronbach, constituiu a base sobre a qual as dimensões e os itens correspondentes foram construídos. Com base nisso, os itens foram cuidadosamente avaliados e mantidos na subescala original, se considerados apropriados, ou movidos para outra dimensão, se fosse mais adequado. Além disso, o alfa de Cronbach foi calculado para cada dimensão e os itens foram excluídos um a cada momento para determinar se a exclusão do item aumentaria o alfa de Cronbach. Com uma avaliação baseada na experiência clínica dos autores, garantindo a validade do conteúdo, os itens foram finalmente colocados na dimensão mais adequada ou excluídos. $\mathrm{O}$ alfa de Cronbach também foi usado para medir a consistência interna (confiabilidade) dos itens. A análise estatística foi realizada usando software SPSS, versão 20.

\section{Fase 3}

A segunda versão da escala foi digitada em um formulário de enquetes do Facebook, publicado na linha do tempo de profissionais que trabalham como autismo e no grupo do da linha de pesquisa (o grupo reúne profissionais e pais de indivíduos com TEA). Foi solicitado novamente que pais de pessoas com ou sem TEA respondessem ao questionário, após lerem e aceitarem o termo de consentimento. Os procedimentos de análise fatorial foram os mesmos já descritos anteriormente.

O projeto "Construção e validação de uma escala para avaliar o comportamento alimentar de crianças com transtornos do espectro do autismo (TEA)" foi aprovado pelo comitê de ética da Escola Bahiana de Medicina e Saúde Pública, por meio do Parecer no 033409/2015.

\section{RESULTADOS}

\section{Fase 1: primeira versão}

A revisão da literatura sobre os instrumentos já existentes para avaliar o padrão alimentar de pessoas com TEA, as entrevistas semiestruturadas com as mães de pessoas com TEA e a experiência clínica dos autores (uma nutricionista, um psiquiatra com especialização e 15 anos de experiência clí- 
nica com pacientes com TEA e um psicólogo especialista em avaliação neuropsicológica) serviram como ponto de partida para a elaboração do instrumento.

As entrevistas semiestruturadas com os pais de pessoas com TEA indicaram quatro dimensões relacionadas ao comportamento alimentar. A primeira foi o "padrão alimentar do indivíduo com TEA", que incluiu problemas na mastigação e seletividade alimentar (preferência, restrição e textura). A segunda foi a "atitude da família frente à alimentação", que incluiu as preferências da família e as suas atitudes relativamente a novos alimentos e à recusa da criança. A terceira foi o "comportamento da criança diante do alimento": alimento usado como moeda de troca, alimentos que modificam o comportamento da criança e estratégia para conseguir o alimento. Houve ainda referência a "problemas gastrointestinais", como refluxo e funcionamento intestinal, relacionados a determinados alimentos. Os resultados desse estudo estão detalhados em outra publicação de Lázaro e Pondé ${ }^{8}$ e indicam que fatores orgânicos (como dificuldade de sucção e de mastigação) e ambientais são determinantes e podem reforçar a seletividade alimentar ou favorecer uma alimentação mais saudável e diversificada.

A partir da revisão da literatura, das entrevistas semiestruturadas e da experiência clínica dos autores da escala, foram estabelecidas seis dimensões do comportamento alimentar, que estão elencadas no quadro 1, bem como as suas definições operacionais e os seus respectivos descritores. A primeira versão da escala foi elaborada com 53 itens, distribuídos nas seis dimensões: Motricidade na Mastigação, Seletividade Alimentar, Aspectos Comportamentais, Sintomas Gastrointestinais, Sensibilidade Sensorial e Habilidades nas Refeições. Na dimensão Motricidade na Mastigação, os itens construídos foram: (1) Dificuldades para mastigar os alimentos; (2) Dificuldade para abrir totalmente a boca; (3) Mastiga os alimentos com a boca aberta; (4) Dificuldade para levar o alimento de um lado para o outro da boca com a língua; (5) Dificuldades ao sugar o peito ou alimentos líquidos usando canudo ou mamadeira; (6) Engole os alimentos sem mastigá-los o bastante; (7) Engasga com os alimentos; (8) Após engolir o alimento, continua com restos de comida na boca; não esvazia totalmente a boca; (9) Precisa beber um líquido para ajudar a engolir a comida; (10) Após engolir a comida, levanta a cabeça para cima como se estivesse ajudando a comida a descer; (11) Durante ou imediatamente após as refeições, golfa (trazendo de volta o alimento que engoliu à boca) e mastiga o alimento novamente. $\mathrm{Na}$ dimensão Seletividade Alimentar, os itens foram: (12) Seleciona alimentos pela marca ou embalagem (por exemplo: somente caixa ou saco plástico); (13) Seleciona alimentos pela temperatura (só quente ou só frio); (14) Seleciona alimentos pela cor; (15) Seleciona o alimento ou rejeita em função da consistência (por exemplo: somente alimento pastoso ou somente líquido ou nenhum dos dois); (16) Seletivo por refeições molhadas (por exemplo: alimentos com molhos ou caldo de feijão); (17) Seletivo por refeições mais secas (por exemplo: sem nenhum molho ou caldo de feijão); (18) Seletivo por alimentos crocantes (por exemplo: batatinha, pão torrado, salgadinho, biscoito cream craker); (19) Seletivo por alimentos com consistência macia (por exemplo: purê, mingau, vitamina, iogurte); (20) Seletivo por alimentos amassados; (21) Seletivo por alimentos liquidificados ou coados no pano ou na peneira; (22) Evita comer carnes; (23) Evita comer frango; (24) Evita comer frutas; (25) Evita comer vegetais cozidos e/ou crus; (26) Retira o tempero da comida (por exemplo: pedaços de coentro, cebolinha ou tomate). Na dimensão Aspectos Comportamentais, os itens foram: (27) Cospe a comida; (28) Possui ritual para comer (por exemplo: os alimentos devem ser arrumados no prato da mesma forma; se o ritual não for obedecido, seu filho se recusa a comer ou fica irritado ou perturbado); (29) Come sempre no mesmo lugar; (30) Come sempre com os mesmos utensílios (por exemplo: o mesmo prato, garfo, colher ou copo); (31) Possui comportamento agressivo durante as refeições (por exemplo: agride quem está por perto, fica se machucando, batendo a cabeça na parede ou se batendo ou beliscando o corpo, destrói objetos); (32) Come uma grande quantidade de alimento num período de tempo curto; (33) Sem permissão, pega a comida de outras pessoas durante as refeições; (34) Sem permissão, pega a comida fora do horário das refeições; (35) Vomita durante ou imediatamente após as refeições; (36) Possui inquietação/agitação motora que dificulta sentar-se à mesa; (37) Bebe, come, lambe substâncias ou objetos estranhos (por exemplo: sabão, terra, plástico, chiclete). Na dimensão

Sintomas Gastrointestinais, os itens foram: (38) Refluxo, golfada (retorno à boca do alimento que comeu ou bebeu); (39) Constipação, intestino preso, ressecado, prisão de ventre; (40) Diarreia; (41) Vômito; (42) Gases; inchaço na barriga; (43) Alergia alimentar (por exemplo: amendoim, frutos do mar); (44) Intolerância ao glúten (o glúten está presente na farinha de trigo, aveia, centeio e cevada); (45) Intolerância à lactose. Na dimensão Sensibilidade Sensorial, os itens foram: (46) Incomoda-se com barulhos (por exemplo: som ou voz alta, liquidificador, carro, moto, maquita etc.); (47) Incomoda-se com cheiros fortes (por exemplo: comida, gasolina, tinta, perfume etc.); (48) Incomoda-se com coisas pegajosas (por exemplo: hidratante, tinta, massa de modelar, beijo molhado etc.); (49) Incomoda-se em ser tocado por outras pessoas (por exemplo: abraço, beijo ou simples toque); (50) Incomoda-se em trocar de roupas, tomar banho, etiqueta e costura nas roupas. Na dimensão Habilidades nas Refeições, os itens foram: (51) Tem dificuldades de sentar-se à mesa para fazer as refeições (por exemplo: almoça no chão, sofá, cama); (52) Derrama muito a comida na mesa ou na roupa quando se alimenta; (53) Tem dificuldades de utilizar os talheres e outros utensílios. 
Optou-se por uma escala de resposta quantitativa com cinco respostas numéricas, ou seja, uma escala tipo Likert, que consiste na medida com categorias de respostas que vão desde o extremo grau de discordância (0) até o extremo de concordância (4) ${ }^{13}$.

\section{Fase 2}

A primeira versão de 53 itens foi submedida à análise da validade do conteúdo pela equipe multidisciplinar de avaliadores. No geral, os itens obtiveram média de 95\% de concordância relativa ao domínio, ao construto, e pequenas alterações foram realizadas na redação de alguns itens por sugestão dos avaliadores. Em seguida, o ajuste semântico foi feito com a aplicação da escala à população-alvo, que foram 20 pais. Alguns itens não foram bem compreendidos pelos familiares e sofreram modificações importantes na sua estrutura. Termos técnicos foram substituídos e frases ambíguas e questões vagas foram reformuladas a fim de que fosse aprimorada a compreensão de todos os itens.

Após esses ajustes, a primeira versão da escala foi postada no Facebook e respondida por 298 pessoas. Foi feita uma análise dos componentes principais com uma rotação Varimax. Foi identificado um total de 13 fatores. O Fator 1 foi composto pelos itens: 1, 2, 3, 4, 5, 6, 7, 8 e 49. O Fator 2, pelos itens: 15, 17, 18, 24, 25 e 26. O Fator 3, pelos itens 36, 37, 51, 52 e 53. O Fator 4, pelos itens 11, 31, 35, 38 e 41. O Fator 5, pelos itens 16, 19, 20 e 21. O Fator 6, pelos itens 28, 29 e 30. O Fator 7, pelos itens 32, 33 e 34. O Fator 8, pelos itens 43, 44 e 45; apesar de formarem um fator, os itens não se correlacionaram bem com os outros itens da escala, sugerindo que fossem retirados. Fator 9, pelos itens 12, 13 e 14. Fator 10, pelos itens 22 e 23. Fator 11, pelos itens 9 e 10. Fator 12, pelos os itens 42 e 40. Fator 13, pelo item 39, que ficou isolado.
Alguns itens não se agruparam em nenhum dos fatores, sendo sugerido retirar: 27, 46, 47, 48 e 50 .

Dos 53 itens inicialmente desenvolvidos para o estudo do construto, 69\% mostraram-se particularmente válidos para a avaliação do atributo. Os 31\% restantes foram eliminados tendo por base os seguintes critérios ${ }^{14,15}$ : 1) o valor absoluto da carga fatorial principal do item foi menor do que 0,40 em qualquer um dos fatores ou assumiu valores negativos; 2) houve ausência de similaridade entre o conteúdo do item e o domínio teórico do construto. Durante o processo, todas essas alternativas foram cuidadosamente avaliadas e consideradas ao decidir itens e a sua localização nas respectivas dimensões.

A dimensão Aspectos Comportamentais, composta incialmente por 11 itens, foi desmembrada ao ser observado que nove itens se agruparam em três fatores de forma a detalhar o tipo de comportamento. Decorrente desse fato e baseando-se na literatura, foram criadas três novas dimensões, sendo elas: Comportamentos Rígidos relacionados à Alimentação (Fator 6 - itens 28, 29 e 30), Comportamento Opositor relacionado à Alimentação (Fator 7 - itens 34, 33 e 32) e Comportamento Inadequado relacionado às Refeições (Fator 4 - itens 31, 11, 41, 35 e 38). Dos três itens restantes da dimensão Aspectos Comportamentais, um item foi eliminado por não ter se agrupado a nada e dois itens se agruparam na dimensão Habilidades nas Refeições (Fator 3 - itens 52, 53, 37, 36 e 51).

A dimensão Motricidade na Mastigação (Fator 1 itens $\mathbf{4}, \mathbf{1}$, 2, 6, 7, 5, 49, 3 e 8) foi a que menos se alterou pelo fato de oito dos 11 itens terem se agrupado. Dois itens ficaram isolados e foram retirados, e o item 11 se agrupou no fator relacionado à nova dimensão Comportamento Inadequado relacionado às Refeições.

Quadro 1. Definição das dimensões da escala e os seus respectivos descritores

\begin{tabular}{|c|c|c|}
\hline Dimensão & Definição & Descritores \\
\hline $\begin{array}{l}\text { Motricidade na } \\
\text { Mastigação }\end{array}$ & $\begin{array}{l}\text { Problemas ligados ao processo de mastigação, sucção e } \\
\text { deglutição dos alimentos }\end{array}$ & $\begin{array}{l}\text { - Dificuldades na mastigação dos alimentos e na sucção de líquidos. } \\
\text { - Engasga ou engole os alimentos sem mastigar. } \\
\text { - Regurgita ou rumina os alimentos, durante ou imediatamente após as refeições. }\end{array}$ \\
\hline $\begin{array}{l}\text { Seletividade } \\
\text { Alimentar }\end{array}$ & $\begin{array}{l}\text { Problemas na diversificação alimentar, caracterizados pela } \\
\text { ingestão restrita de tipos de alimentos }\end{array}$ & $\begin{array}{l}\text { - Seleção por preparações ou alimentos pela marca, embalagem, temperatura, cor ou textura. } \\
\text { - Seleção por preparações, ou pelo fato de os alimentos serem secos ou úmidos. } \\
\text { - Seleção pela forma de processamento do alimento, podendo ser amassado, liquidificado, } \\
\text { liquidificado e coado. } \\
\text { - Seleção por tipo de alimento: carnes, frango, vegetais, frutas ou temperos. }\end{array}$ \\
\hline $\begin{array}{l}\text { Aspectos } \\
\text { Comportamentais }\end{array}$ & $\begin{array}{l}\text { Problemas manifestados ou decorrentes no período das } \\
\text { refeições ou relacionados à ingestão }\end{array}$ & $\begin{array}{l}\text { - Presença de rituais durante as refeições. } \\
\text { - Manifestação de comportamentos disruptivos e/ou agressivos. } \\
\text { - Necessidade de ingerir objetos estranhos que não são alimentos (PICA). }\end{array}$ \\
\hline $\begin{array}{l}\text { Sintomas } \\
\text { Gastrointestinais }\end{array}$ & $\begin{array}{l}\text { Problemas relacionados ao trato gastrointestinal sem a } \\
\text { presença de uma doença base }\end{array}$ & $\begin{array}{l}\text { - Refluxo e/ou vômito. } \\
\text { - Presença de alterações intestinais. } \\
\text { - Alergia alimentar. } \\
\text { - Intolerância ao glúten e/ou à lactose. }\end{array}$ \\
\hline $\begin{array}{l}\text { Sensibilidade } \\
\text { Sensorial }\end{array}$ & $\begin{array}{l}\text { Presença de disfunção sensorial relacionada ao } \\
\text { processamento sensorial }\end{array}$ & - Presença de hipo ou hipersensibilidade auditiva, olfativa ou tátil. \\
\hline $\begin{array}{l}\text { Habilidades nas } \\
\text { Refeições }\end{array}$ & $\begin{array}{l}\text { Dificuldades relacionadas ao ato de alimentar-se de forma } \\
\text { independente }\end{array}$ & - Ausência de habilidades no manuseio de talheres e objetos, principalmente durante as refeições. \\
\hline
\end{tabular}


Com relação à Seletividade Alimentar (Fator 2 - itens

$\mathbf{2 4}, \mathbf{1 5}, \mathbf{2 6}, \mathbf{2 5}, 17$ e $\mathbf{1 8}$ ), composta na teoria por 15 itens, sete itens se agruparam e permaneceram nessa dimensão, enquanto oito foram eliminados: um por ter se isolado e sete por se agruparem em três fatores distintos, de modo que, do ponto de vista clínico, não fez sentido mantê-los.

$\mathrm{Na}$ dimensão Sintomas Gastrointestinais (Fator 8 itens 43, 44 e 45), dos oito fatores, apenas três permaneceram. Os outros cinco fatores foram agrupados em dimensões que não apresentaram embasamento teórico.

A dimensão Sensibilidade Sensorial foi totalmente excluída pelo fato de quatro itens $(46,47,48$ e 50$)$ não se correlacionarem com nada. Foram eliminados os fatores: 5 (itens - 20, 21, 19 e 16), 9 (itens - 12, 13 e 14), 10 (itens - 22 e 23), 11 (itens - 10 e 9) e 12 (itens - 42 e 40).

Após as análises pertinentes, os autores se reuniram e todas as dimensões foram reavaliadas baseando-se em dados científicos da literatura, sendo construída uma segunda versão da escala. Assim, dos 53 itens da primeira versão da escala, foram eliminados 20, com base na análise fatorial, e acrescentados três, por consenso dos autores, em consonância com a teoria: Quer comer alimentos sempre da mesma marca, embalagem ou personagem (por exemplo: bebe suco somente de caixinha, quer somente produtos do Bob Esponja); Quer comer sempre os mesmos alimentos (por exemplo: se comeu frango hoje, quer amanhã novamente) e Quer comer alimentos com cor semelhante (por exemplo: somente sucos amarelos - manga, maracujá, laranja). A segunda versão da escala foi composta, portanto, por com 35 itens.

\section{Fase 3: validação interna, construção dos itens da escala}

A segunda versão da escala foi respondida via Facebook por 130 pessoas, das quais 90 tinham filho com diagnóstico de TEA. Dos 35 itens que permaneceram após a primeira análise fatorial, 26 mostraram-se particularmente válidos para a avaliação do atributo. Os 25\% restantes foram eliminados, tendo por base os seguintes critérios ${ }^{14,16}: 1$ ) o valor absoluto da carga fatorial principal do item foi menor do que 0,40 em qualquer um dos fatores (três itens) ou assumiu valores negativos (dois itens); 2) houve ausência de similaridade entre o conteúdo do item e o domínio teórico do construto (três itens).

Considerando os 26 itens resultantes da análise fatorial, as dimensões do comportamento alimentar encontram-se dispostas na seguinte ordem: 1) Motricidade na Mastigação; 2) Seletividade Alimentar; 3) Habilidades nas Refeições; 4) Comportamento Inadequado relacionado às Refeições;

5) Comportamentos Rígidos relacionados à Alimentação;

6) Comportamento Opositor relacionado à Alimentação; 7) Alergias e Intolerância Alimentar. Uma vez garantida a validade de construto do instrumento pelo método da análise fatorial, procedeu-se ao exame da fidedignidade das dimensões e da escala como um todo, pelo cálculo do coeficiente alfa de Cronbach. Considerando que o alfa não deve apresentar um número inferior a 0,70, o que mostraria um baixo nível de consistência interna entre os itens de uma mesma dimensão, os sete fatores avaliados exibiram valores satisfatórios: 1) Motricidade na Mastigação $(0,779)$; 2$)$ Seletividade Alimentar $(0,806)$; 3) Habilidades nas Refeições $(0,832)$; 4) Comportamento Inadequado relacionado às Refeições $(0,774)$; 5) Comportamentos Rígidos relacionados à Alimentação $(0,828) ; 6)$ Comportamento Opositor relacionado à Alimentação $(0,700)$; 7$)$ Alergias e Intolerância Alimentar $(0,789)$. A estrutura final da escala ficou composta por 26 itens, distribuídos em sete fatores, apresentando um valor geral de confiabilidade de 0,867 (Tabela 1).

\section{DISCUSSÃO}

A Escala para Avaliação do Comportamento Alimentar em pessoas com TEA apresentou adequados índices psicométricos para o estudo de validade. O novo instrumentos traz aspetos inovadores ao propor a primeira escala de avaliação do comportamento alimentar especificamente para crianças com TEA, em língua portuguesa.

O Brief Autism Mealtime Behavior Inventory (BAMBI) ${ }^{17}$ foi o primeiro instrumento desenvolvido especificamente para avaliar os problemas alimentares de crianças com autismo. Esse instrumento teve como foco os aspectos comportamentais relacionados à alimentação, deixando de explorar os problemas sensoriais e gastrointestinais frequentemente encontrados no TEA e que poderiam influenciar o comportamento alimentar. O Screening Tool for Feeding Problems (STEP- CHILD) ${ }^{18}$, além dos aspectos comportamentais, incluiu itens relativos a seletividade alimentar, problemas na motricidade oral, habilidades ligadas à alimentação e recusa alimentar. Esses elementos permitiram mapear outros fatores intervenientes na construção da dinâmica alimentar de indivíduos autistas. O STEP-CHILD ${ }^{18}$ trouxe importante contribuição para a literatura cientifica ao sugerir a associação entre características das crianças [peso, índice de massa corporal (IMC), problemas alimentares] e variáveis relacionadas aos pais, como atitude dos pais durante as refeições. A atitude excessivamente permissiva dos pais, a exemplo da pouca insistência para comer durante as refeições, ou a frequente preparação de refeições especiais diferente das dos demais membros da família também parecem influenciar no perfil alimentar de crianças com TEA $A^{8,18}$. Pais que apresentam sobrepeso e possuem problemas no controle da própria ingestão de alimentos $^{19}$ influenciam negativamente o hábito alimentar dos fiIhos ${ }^{20-22}$. Outra contribuição do STEP-CHILD ${ }^{18}$ foi a introdução do item "roubar comida" como aspecto comportamental do TEA e que anteriormente só havia sido descrito em adultos com necessidades especiais ${ }^{18}$. Algumas limitações devem ser salientadas no STEP-CHILD'8: a escala não explorou os 
Tabela 1. Carga fatorial associada a cada item da escala

\begin{tabular}{|c|c|c|c|c|c|c|c|}
\hline & F1 & F2 & F3 & F4 & F5 & $\mathrm{F} 6$ & F7 \\
\hline Dificuldades para mastigar os alimentos & 0,780 & & & & & & \\
\hline Engole os alimentos sem mastigá-los o bastante & 0,770 & & & & & & \\
\hline Dificuldade para levar 0 alimento de um lado para 0 outro da boca com a língua & 0,689 & & & & & & \\
\hline Mastiga os alimentos com a boca aberta & 0,635 & & & & & & \\
\hline Evita comer vegetais cozidos e/ou crus & & 0,895 & & & & & \\
\hline Retira o tempero da comida (ex.: pedaços de coentro, cebolinha ou tomate) & & 0,771 & & & & & \\
\hline Evita comer frutas & & 0,748 & & & & & \\
\hline Possui inquietação/agitação motora que dificulta sentar-se à mesa & & & 0,831 & & & & \\
\hline Tem dificuldades de sentar-se à mesa para fazer as refeições (ex.: almoça no chão, sofá, cama) & & & 0,751 & & & & \\
\hline Tem dificuldades de utilizar os talheres e outros utensílios & & & 0,695 & & & & \\
\hline Derrama muito a comida na mesa ou na roupa quando se alimenta & & & 0,655 & & & & \\
\hline Bebe, come, lambe substâncias ou objetos estranhos (ex.: sabão, terra, plástico, chiclete) & & & 0,636 & & & & \\
\hline Vomita, durante ou imediatamente após as refeições & & & & 0,850 & & & \\
\hline $\begin{array}{l}\text { Durante ou imediatamente após as refeições, golfa (trazendo de volta } 0 \text { alimento que engoliu à boca) e mastiga } \\
0 \text { alimento novamente }\end{array}$ & & & & 0,809 & & & \\
\hline Come sempre com os mesmos utensílios (ex.: o mesmo prato, garfo, colher ou copo) & & & & & 0,779 & & \\
\hline Come sempre no mesmo lugar & & & & & 0,717 & & \\
\hline Quer comer sempre os mesmos alimentos (ex.: se comeu frango hoje, quer amanhã novamente) & & & & & 0,700 & & \\
\hline Quer comer alimentos com cor semelhante (ex.: somente quer sucos amarelos - manga, maracujá, laranja) & & & & & 0,700 & & \\
\hline $\begin{array}{l}\text { Quer comer alimentos sempre da mesma marca, embalagem ou personagem (ex.: bebe suco somente de } \\
\text { caixinha, quer somente produtos do Bob Esponja) }\end{array}$ & & & & & 0,666 & & \\
\hline $\begin{array}{l}\text { Possui ritual para comer (ex.: os alimentos devem ser arrumados no prato da mesma forma; se o ritual não for } \\
\text { obedecido, seu filho se recusa a comer ou fica irritado ou perturbado) }\end{array}$ & & & & & 0,650 & & \\
\hline Sem permissão, pega a comida fora do horário das refeições & & & & & & 0,853 & \\
\hline Sem permissão, pega a comida de outras pessoas durante as refeições & & & & & & 0,723 & \\
\hline Come uma grande quantidade de alimento num período de tempo curto & & & & & & 0,616 & \\
\hline Intolerância ao glúten (o glúten está presente na farinha de trigo, aveia, centeio e cevada) & & & & & & & 0,893 \\
\hline Alergia alimentar (ex.: amendoim, frutos do mar) & & & & & & & 0,798 \\
\hline Tem intolerância à lactose & & & & & & & 0,757 \\
\hline
\end{tabular}

problemas sensoriais e gastrointestinais e os comportamentos ritualísticos característicos do transtorno e que podem influenciar no padrão alimentar. O SWedish Eating Assessment for Autism spectrum disorders (SWEAA) ${ }^{23}$, apesar de ter demonstrado boa validade e confiabilidade interna baseada nos parâmetros estatísticos, não contemplou a heterogeneidade do transtorno. Ao estabelecer ponto de corte em função do quociente de inteligência (QI) dos participantes, a escala foi testada predominantemente (72\%) em autistas com alto rendimento e com síndrome de Asperger. Com relação aos fatores avaliados pelo conteúdo da escala, importantes tópicos ligados ao comportamento alimentar foram abordados nesse instrumento, a exemplo do controle motor, ambiente das refeições, comportamentos ritualísticos, aspectos sociais no momento das refeições e alguns distúrbios alimentares como o desejo de ingerir coisas que não são consideradas alimentos (PICA). A SWEAA ${ }^{23}$, no entanto, não aborda os distúrbios gastrointestinais, importante aspecto associado aos problemas alimentares em indivíduos com TEA. O Behavioral
Pediatrics Feeding Assessment Scale (BPFAS)24 é uma medida abrangente e amplamente utilizada para detectar problemas de alimentação e comportamentais. Trata-se de medida válida, confiável e que discrimina de forma eficaz as crianças com problemas de alimentação em populações com desenvolvimento típico e com questões clínicas envolvidas. Mesmo sem ter passado por um devido processo de validação, o BPFAS24 já havia sido utilizado em pesquisas envolvendo o TEA ${ }^{17,25}$ e em alguns casos como instrumento de avaliação do construto de outras escalas. Durante os procedimentos de validação e verificação da estrutura do BPFAS $^{24}$ em uma amostra com TEA ${ }^{26}$, foi observada a inadequação da estrutura de cinco fatores dessa escala, e os fatores "recusa de alimentos com textura" (a Cronbach $=0,26$ ) e "recusa geral da criança mais velha" (a Cronbach $=0,3$ ) demonstraram baixa consistência interna. O mesmo ocorreu em outros estudos nos quais a amostra de crianças possuía o diagnóstico de doença crônica ou estava com sobrepeso ou obesidade ${ }^{27}$. Apesar de ter sido desenvolvido para avaliar o problema alimentar de 
crianças de 1 a 8 anos de idade, o BPFAS ${ }^{24}$ não levou em conta características específicas do TEA, a exemplo das questões sensoriais e dos distúrbios gastrointestinais.

Em comparação a outros instrumentos internacionais, é possível avaliar aspectos específicos do TEA como a escala $\mathrm{BAMBI}^{17}$ e os aspectos da seletividade alimentar e motricidade oral com o STEP-CHILD ${ }^{18}$. Avança ao englobar os aspectos gastrointestinais e problemas sensoriais que outras escalas não avaliavam. Contempla a avaliação do fator relacionado a aspectos comportamentais como na escala BPFAS ${ }^{24}$. O novo instrumento desenvolvido engloba vários elementos acima citados que apresentavam falhas ao avaliar crianças com TEA, tentando apresentar um instrumento mais completo para a avaliação do comportamento alimentar de pessoas com TEA. Futuramente, com a aplicação desse instrumento, os indivíduos poderão ser agrupados por fenótipos para meIhor compreensão dos fatores intervenientes dos problemas relacionados ao comportamento alimentar.

\section{CONCLUSÃO}

Fica então disponível a Escala para Avaliação do Comportamento Alimentar em pessoas com TEA (Anexo 1), com o objetivo de identificar, de forma detalhada, na clínica, as perturbações alimentares em pessoas com TEA, proporcionando um encaminhamento mais específico em relação às suas necessidades. O propósito da escala é identificar as dimensões do comportamento alimentar que estão alteradas, para que possa haver um direcionamento mais específico em relação à terapêutica, bem como ser um instrumento para mensurar a evolução do tratamento.

\section{CONTRIBUIÇÕES INDIVIDUAIS}

Cristiane Lázaro - Construção dos itens, referencial teórico e coleta de dados.

Gustavo Siquara - Análises estatísticas, interpretação dos dados e auxílio na construção dos itens.

Milena Pondé - Orientadora do estudo, coleta de dados, concepção e desenho do estudo.

\section{CONFLITO DE INTERESSES}

Os autores do presente trabalho não possuem conflito de interesses a serem declarados.

\section{REFERÊNCIAS}

1. Sacrey LA, Germani T, Bryson SE, Zwaigenbaum L. Reaching and grasping in autism spectrum disorder: a review of recent literature. Front Neurol. 2014;5:6.
2. Chaidez V, Hansen RL, Hertz-Picciotto I. Gastrointestinal problems in children with autism, developmental delays or typical development. J Autism Dev Disord. 2014;44(5):1117-27.

3. Cermak SA, Curtin C, Bandini LG. Food selectivity and sensory sensitivity in children with autism spectrum disorders. J Am Diet Assoc. 2010;110(2):238-46.

4. Green SA, Ben-Sasson A. Anxiety disorders and sensory over-responsivity in children with autism spectrum disorders: Is there a causal relationship? J Autism Dev Disord. 2010:40(12):1495-504.

5. American Psychiatric Association. Diagnostic and Statistical Manual of Mental Disorders (DSM-IV-TR). 4th ed. Washington, DC: American Psychiatric Association; 2004.

6. American Psychiatric Association. Diagnostic and Statistical Manual of Mental Disorders (DSM-V). 5th ed. Washington, DC: American Psychiatric Association; 2013.

7. Lázaro CP, Caron J, Pondé MP. Escalas de avaliação do comportamento alimentar de indivíduos com transtorno do espectro autista. Psicol Teor Prat. 2018;20(3):23-41.

8. Lázaro CP, Pondé MP. Narratives of mothers of children with autism spectrum disorders: focus on eating behavior. Trends Psychiatry Psychother. 2017;39(3):180-7.

9. Brace I. Questionnaire design: how to plan, structure and write survey material for effective market research. London: Kogan Page; 2004.

10. Urbina S. Fundamentos da testagem psicológica. Porto Alegre: Artmed; 2007.

11. Pasquali L. Validade dos testes psicológicos: será possível reencontrar o caminho? Psicol Teor Pesq. 2007;23:99-107.

12. Fayers PM, Machin D. Scores and measurements: validity, reliability, sensitivity. In: Fayers PM, Machin D, eds. Quality of Life: the assessment, analysis and interpretation of patientreported outcomes. 2nd ed. Chichester: John Wiley \& Sons; 2007. p. 77-108.

13. Dalmoro M, Vieira KM. Dilemas na construção de escalas Tipo Likert: 0 número de itens e a disposição influenciam nos resultados? Rev Gestão Org. 2013;6(3):161-74.

14. Gorsuch RL. Factor analysis. Handbook of Psychology. Part One. Wiley Online Library; 2003. p. 143-64.

15. Smith GT, Mccarthy DM. Methodological considerations in the refinement of clinical assessment instruments. Psychol Assess. 1995;7(3):300-8.

16. Tabachnick BG, Fidell LS. Using multivariate statistics. 3rd ed. New York: Harper Collins; 1996.

17. Lukens CT, Linscheid TR. Development and validation of an inventory to assess mealtime behavior problems in children with autism. J Autism Dev Disord. 2008;38(2):342-52.

18. Seiverling L, Hendy HM, Williams K. The Screening Tool of Feeding Problems applied to children (STEP-CHILD): psychometric characteristics and associations with child and parent variables. Res Dev Disabil. 2011;32(3):1122-9.

19. Birch $L L$, Davison KK. Family environmental factors influencing the developing behavioral controls of food intake and childhood overweight. Pediatr Clin North Am. 2001;48(4):893-907.

20. Hendrie G, Sohonpal G, Lange K, Golley R. Change in the family food environment is associated with positive dietary change in children. Int J Behav Nutr Phys Act. 2013;10:4.

21. Scaglioni S, Arrizza C, Vecchi F, Tedeschi S. Determinants of children's eating behavior. Am J Clin Nutr. 2011;94(6 Suppl):2006S-11S.

22. Scaglioni S, Salvioni M, Galimberti C. Influence of parental attitudes in the development of children eating behaviour. Br J Nutr. 2008;99 Suppl 1:S22-5.

23. Karlsson L, Råstam M, Wentz E. The SWedish Eating Assessment for Autism spectrum disorders (SWEAA)-Validation of a self-report questionnaire targeting eating disturbances within the autism spectrum. Res Dev Disabil. 2013;34(7):2224-33.

24. Crist W, Napier-Phillips A. Mealtime behaviors of young children: a comparison of normative and clinical data. J Dev Behav Pediatr. 2001;22(5):279-86.

25. Martins Y, Young RL, Robson DC. Feeding and eating behaviors in children with autism and typically developing children. J Autism Dev Disord. 2008;38(10):1878-87.

26. Allen SL, Smith IM, Duku E, Vaillancourt T, Szatmari P, Bryson S, et al. Behavioral Pediatrics Feeding Assessment Scale in young children with autism spectrum disorder: psychometrics and associations with child and parent variables. J Pediatr Psychol. 2015;40(6):581-90.

27. Davis AM, Canter KS, Stough CO, Gillette MD, Patton S. Measurement of mealtime behaviors in rural overweight children: an exploratory factor analysis of the Behavioral Pediatrics Feeding Assessment Scale. J Pediatr Psychol. 2014;39(3):332-9. 
Anexo 1. Escala LABIRINTO de Avaliação do Comportamento Alimentar no TEA

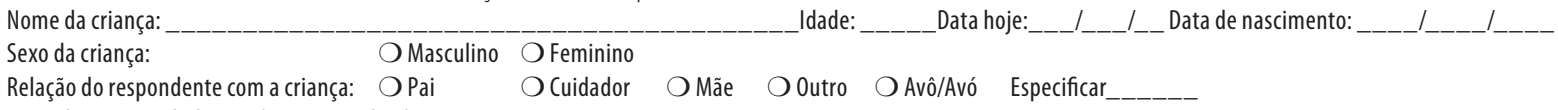

Formulário preenchido por: (nome completo)

Por favor, preencha este questionário de acordo com a sua opinião sobre o comportamento alimentar do seu filho, mesmo que a sua opinião seja diferente daquela de outras pessoas. Caso tenha algum comentário adicional, pode anotar ao lado de cada item ou no final do questionário. POR FAVOR, RESPONDA A TODOS OS ITENS.

Abaixo há uma lista de vários problemas ou dificuldades relacionadas ao comportamento alimentar. As opções de resposta variam de 1 (Não) até 5 (Sempre). Coloque um círculo em torno da resposta que mais se adéqua à criança:

1. Não: Se seu filho(a) não apresenta o comportamento (nunca);

2. Raramente: Se seu filho(a) raramente apresenta o comportamento descrito;

3. Às vezes: Se seu filho(a) às vezes apresenta 0 comportamento;

4. Frequentemente: Se seu filho(a) com frequência apresenta o comportamento;

5. Sempre: Se seu filho(a) sempre apresenta o comportamento.

Escala LABIRINTO de Avaliação do Comportamento Alimentar no TEA

1. Dificuldades para mastigar os alimentos

2. Engole os alimentos sem mastigá-los 0 bastante

3. Dificuldade para levar 0 alimento de um lado para o outro da boca com a língua

4. Mastiga os alimentos com a boca aberta

5. Evita comer vegetais cozidos e/ou crus

6. Retira o tempero da comida (ex.: pedaços de coentro, cebolinha ou tomate)

7. Evita comer frutas

8. Possui inquietação/agitação motora que dificulta sentar-se à mesa

9. Tem dificuldades de sentar-se à mesa para fazer as refeições (ex.: almoça no chão, sofá, cama)

10. Tem dificuldades de utilizar os talheres e outros utensílios

11. Derrama muito a comida na mesa ou na roupa quando se alimenta

12. Bebe, come, lambe substâncias ou objetos estranhos (ex.: sabão, terra, plástico, chiclete)

13. Vomita, durante ou imediatamente após as refeições

14. Durante ou imediatamente após as refeições, golfa (trazendo de volta 0 alimento que engoliu à boca) e mastiga 0 alimento novamente

15. Come sempre com os mesmos utensílios (ex.: o mesmo prato, garfo, colher ou copo)

16. Come sempre no mesmo lugar

17. Quer comer sempre os mesmos alimentos (ex.: se comeu frango hoje, quer amanhã novamente)

18. Quer comer alimentos com cor semelhante (ex.: somente quer sucos amarelos - manga, maracujá, laranja)

19. Quer comer alimentos sempre da mesma marca, embalagem ou personagem (ex.: bebe suco somente de caixinha, quer somente produtos do Bob Esponja)

20. Possui ritual para comer (ex.: os alimentos devem ser arrumados no prato da mesma forma; se o ritual não for obedecido, seu filho se recusa a comer ou fica irritado ou perturbado)

\section{Sem permissão, pega a comida fora do horário das refeições}

22. Sem permissão, pega a comida de outras pessoas durante as refeições

23. Come uma grande quantidade de alimento num período de tempo curto)

24. Intolerância ao glúten (o glúten está presente na farinha de trigo, aveia, centeio e cevada)

25. Alergia alimentar (ex.: amendoim, frutos do mar)

26. Tem intolerância à lactose

\begin{tabular}{|c|c|c|c|c|}
\hline Não & Raramente & Às vezes & Frequentemente & Sempre \\
\hline 0 & 1 & 2 & 3 & 4 \\
\hline 0 & 1 & 2 & 3 & 4 \\
\hline 0 & 1 & 2 & 3 & 4 \\
\hline 0 & 1 & 2 & 3 & 4 \\
\hline 0 & 1 & 2 & 3 & 4 \\
\hline 0 & 1 & 2 & 3 & 4 \\
\hline 0 & 1 & 2 & 3 & 4 \\
\hline 0 & 1 & 2 & 3 & 4 \\
\hline 0 & 1 & 2 & 3 & 4 \\
\hline 0 & 1 & 2 & 3 & 4 \\
\hline 0 & 1 & 2 & 3 & 4 \\
\hline 0 & 1 & 2 & 3 & 4 \\
\hline 0 & 1 & 2 & 3 & 4 \\
\hline 0 & 1 & 2 & 3 & 4 \\
\hline 0 & 1 & 2 & 3 & 4 \\
\hline 0 & 1 & 2 & 3 & 4 \\
\hline 0 & 1 & 2 & 3 & 4 \\
\hline 0 & 1 & 2 & 3 & 4 \\
\hline 0 & 1 & 2 & 3 & 4 \\
\hline 0 & 1 & 2 & 3 & 4 \\
\hline 0 & 1 & 2 & 3 & 4 \\
\hline 0 & 1 & 2 & 3 & 4 \\
\hline 0 & 1 & 2 & 3 & 4 \\
\hline 0 & 1 & 2 & 3 & 4 \\
\hline 0 & 1 & 2 & 3 & 4 \\
\hline 0 & 1 & 2 & 3 & 4 \\
\hline
\end{tabular}

Comentários Adicionais:

Soma dos Fatores

Fatores da Escala

Fator 1: Motricidade na Mastigação

Fator 2: Seletividade Alimentar

Fator 3: Habilidades nas Refeições

Fator 4: Comportamento Inadequado relacionado às Refeições

Fator 5: Comportamentos Rígidos relacionados à Alimentação

Fator 6: Comportamento Opositor relacionado à Alimentação

Fator 7: Alergias e Intolerância Alimentar

\begin{tabular}{|c|c|}
\hline Itens & Total \\
\hline $1: \_2:-33:-4:_{-}$ & - \\
\hline 5:_6:__7:-_ & - \\
\hline 8:__9:__10:_11:__12:_- & - \\
\hline 13:_-_14:_- & - \\
\hline 15:_16:_17:__18:_19:__20:_- & - \\
\hline 21:__22:__ 23:_-_ & - \\
\hline 24:_25:__26:_- & - \\
\hline
\end{tabular}

